\title{
Impact of Time-of-Flight PET on Quantification Errors in MR Imaging-Based Attenuation Correction
}

\author{
Abolfazl Mehranian ${ }^{1}$ and Habib Zaidi ${ }^{1-3}$ \\ ${ }^{1}$ Division of Nuclear Medicine and Molecular Imaging, Geneva University Hospital, Geneva, Switzerland; ${ }^{2}$ Department of Nuclear \\ Medicine and Molecular Imaging, University of Groningen, Groningen, Netherlands; and ${ }^{3}$ Geneva Neuroscience Centre, University \\ of Geneva, Geneva, Switzerland
}

Time-of-flight (TOF) PET/MR imaging is an emerging imaging technology with great capabilities offered by TOF to improve image quality and lesion detectability. We assessed, for the first time, the impact of TOF image reconstruction on PET quantification errors induced by MR imaging-based attenuation correction (MRAC) using simulation and clinical PET/CT studies. Methods: Standard 4-class attenuation maps were derived by segmentation of CT images of 27 patients undergoing PET/CT examinations into background air, lung, soft-tissue, and fat tissue classes, followed by the assignment of predefined attenuation coefficients to each class. For each patient, 4 PET images were reconstructed: non-TOF and TOF both corrected for attenuation using reference CT-based attenuation correction and the resulting 4-class MRAC maps. The relative errors between non-TOF and TOF MRAC reconstructions were compared with their reference CT-based attenuation correction reconstructions. The bias was locally and globally evaluated using volumes of interest (VOIs) defined on lesions and normal tissues and CT-derived tissue classes containing all voxels in a given tissue, respectively. The impact of TOF on reducing the errors induced by metal-susceptibility and respiratory-phase mismatch artifacts was also evaluated using clinical and simulation studies. Results: Our results show that TOF PET can remarkably reduce attenuation correction artifacts and quantification errors in the lungs and bone tissues. Using classwise analysis, it was found that the non-TOF MRAC method results in an error of $-3.4 \% \pm 11.5 \%$ in the lungs and $-21.8 \% \pm 2.9 \%$ in bones, whereas its TOF counterpart reduced the errors to $-2.9 \% \pm 7.1 \%$ and $-15.3 \% \pm 2.3 \%$, respectively. The VOI-based analysis revealed that the non-TOF and TOF methods resulted in an average overestimation of $7.5 \%$ and $3.9 \%$ in or near lung lesions $(n=23)$ and underestimation of less than $5 \%$ for soft tissue and in or near bone lesions $(n=91)$. Simulation results showed that as TOF resolution improves, artifacts and quantification errors are substantially reduced. Conclusion: TOF PET substantially reduces artifacts and improves significantly the quantitative accuracy of standard MRAC methods. Therefore, MRAC should be less of a concern on future TOF PET/MR scanners with improved timing resolution.

Key Words: TOF; PET/MRI; attenuation correction; quantification; whole-body imaging

J Nucl Med 2015; 56:635-641

DOI: 10.2967/jnumed.114.148817

Received Sep. 22, 2014; revision accepted Jan. 21, 2015.

For correspondence or reprints contact: Habib Zaidi, Geneva University Hospital, 24 rue Micheli-du-Crest, Geneva 1211, Switzerland.

E-mail: habib.zaidi@hcuge.ch.

Published online Mar. 5, 2015.

COPYRIGHT (c) 2015 by the Society of Nuclear Medicine and Molecular Imaging, Inc.
$\mathbf{H}$ dality enabling simultaneous molecular and morphologic assessment of a variety of physiopathologic conditions (1). Over the last 2 decades, PET/MR technology has experienced considerable technical advances toward addressing the challenges encountered in system design and quantitative performance. With the advent of avalanche photodiodes and silicon photomultipliers, the challenge of mutual compatibility between PET and MR subsystems has now been well addressed, thus paving the way toward fully integrated time-of-flight (TOF) PET/MR systems (2). However, accurate PET quantification using MR imaging-based attenuation correction (MRAC) remains a major challenge (3).

MR signals are related to proton density, relaxation time properties of tissues, and the selected pulse parameters. Therefore, there is not a unique mapping between MR intensities and attenuation coefficients at $511 \mathrm{keV}$, as in PET/CT. Moreover, bones cannot be well delineated on conventional MR sequences because of their low water content and short transverse relaxation time. Truncation of MR imaging field of view and metal-induced susceptibility artifacts are other problems encountered in MRAC (4). Generally, MRAC methods can be categorized in 3 groups: segmentation-based approaches, which segment MR images into different tissue classes (background air, lung, fat, and soft tissue) and assign predefined attenuation coefficients to each class (5); atlas registration-based approaches, in which a coregistered MR-CT atlas dataset is used to derive a pseudo CT image from the patient's MR image (6); and emission-based approaches in which the attenuation map is directly estimated from TOF PET emission data with MR anatomic prior information $(7,8)$. Currently, segmentation-based MRAC is the standard approach on commercial PET/MR scanners.

In 3- or 4-class MRAC methods, bones are replaced by soft tissue and the inter- or intrapatient heterogeneity of attenuation coefficients in different tissue classes is ignored, which leads to quantification errors in the estimation of standardized uptake value (SUV) ranging between $4 \%$ and $25 \%$ in different organs (9-13). Using the PET/CT datasets of 35 patients, Martinez-Möller et al. reported an average SUV error of $8.0 \% \pm 3.3 \%$ in 21 bone lesions and less than $5 \%$ in all other lesions (5). Schulz et al. reported, using 15 whole-body PET/CT/MR patient scans, an average error of $6.5 \% \pm 4.1 \%$ in 7 bone lesions and a maximum error of $-13.4 \%$ in a pelvic bone lesion (14). Ouyang et al. demonstrated that if the bones can be identified in MR images to produce a 5-class MRAC map, SUV errors in all bones are reduced to less than $10 \%(10)$. Ultrashort echo time pulse sequences have been extensively explored to include bones as a fifth class and reduce PET quantification 


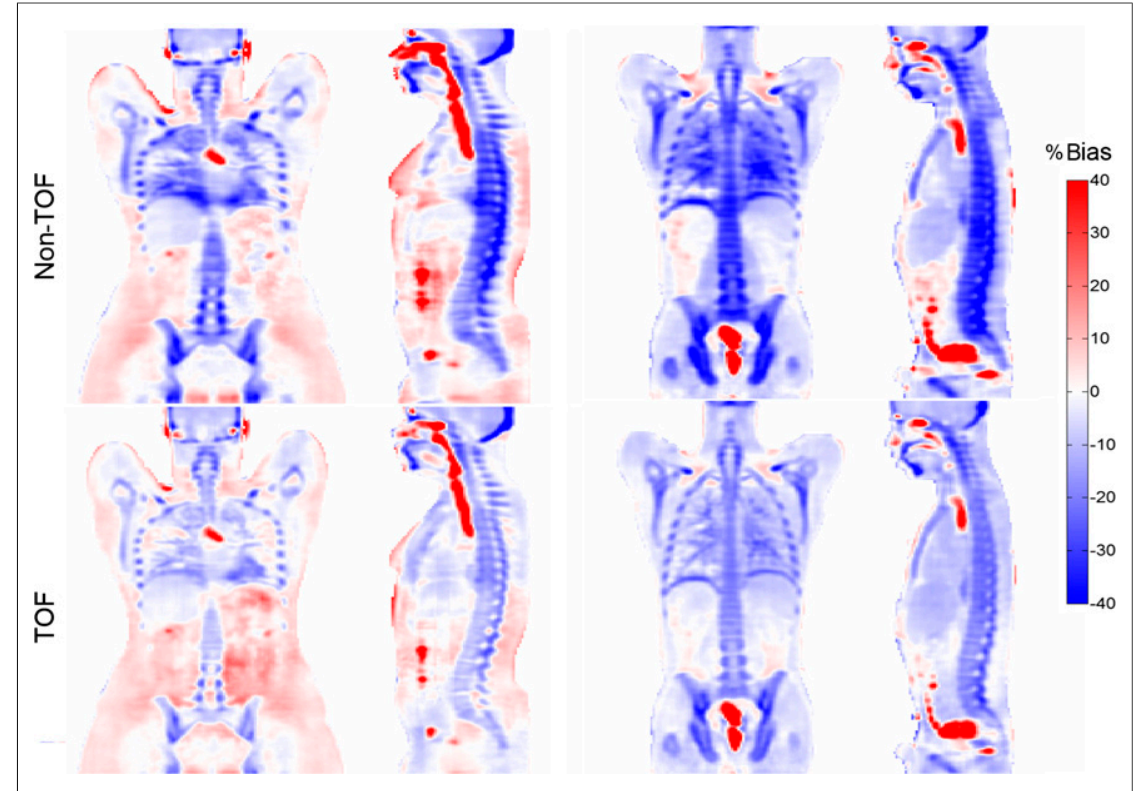

FIGURE 1. Representative bias maps between PET images corrected for attenuation using 4-class MRAC and CTAC techniques obtained using non-TOF and TOF reconstructions for 2 patients with high (left panel) and low (right panel) body mass indexes.

errors (15). Despite the promising results, ultrashort echo time sequences are time-consuming and not yet clinically feasible.

With the advent of clinical TOF PET systems, several studies reported that TOF can improve PET image quality in terms of signalto-noise ratio, lesion detectability, convergence rate, and tolerance to inconsistencies between emission and correction data compared with non-TOF reconstructions (16). Using 2-dimensional phantom simulations, Boellaard et al. showed that TOF PET reconstruction can reduce PET quantification errors when using MR-based attenuation correction (17). In fact, in TOF PET, the difference in arrival times of coincident annihilation photons are measured with an uncertainty governed by the timing resolution of the detectors. During PET image reconstruction, the additional TOF information is exploited to update image voxels only along segments of response, defined by TOF resolution, instead of the whole line of response. Consequently, the cross-dependencies between image voxels are reduced, which results in reduced noise propagation and fast and space-invariant convergence (thus improving the detectability of lesions near large hot regions such as the liver) and reduced sensitivity to errors in normalization, attenuation correction, and scatter correction.

Considering the fast development of TOF PET detectors toward coincidence timing resolutions of less than $100 \mathrm{ps}(18)$ and the lack of clinical studies highlighting the importance of TOF in MRAC PET, we aimed at systematically evaluating the impact of TOF on PET quantification errors induced by 4-class MRAC. In addition, we evaluated the performance of TOF PET in the presence of metal susceptibility and respiratory mismatch artifacts in MRAC maps. To explore the trend of bias reduction in future-generation PET/MR scanners with improved timing resolution, we conduct realistic 3-dimensional simulations using different TOF resolutions in phantoms derived from clinical ${ }^{18} \mathrm{~F}$-FDG examinations.

\section{MATERIALS AND METHODS}

\section{Data Acquisition and Image Reconstruction}

PET/CT datasets were acquired on the Biograph mCT Flow scanner (Siemens Healthcare). The PET subsystem of the scanner consists of 4 rings of 48 detector blocks, covering axial and transaxial fields of view of 218 and $700 \mathrm{~mm}$, respectively. PET data were acquired with an

TABLE 1

PET Quantification Bias of Non-TOF vs. TOF in Different VOls and Tissue Classes

\begin{tabular}{clcc}
\hline \multicolumn{1}{c}{ Parameter } & Location & Non-TOF & TOF \\
\hline Normal regions $(\mathrm{VOI})$ & Lung & $-1.0 \% \pm 16.6 \%(16.7 \%)$ & $-1.5 \% \pm 8.0 \%(8.1 \%)$ \\
& T3/L4 & $-16.8 \% \pm 5.8 \%(17.7 \%)$ & $-8.8 \% \pm 4.9 \%(10.1 \%)$ \\
& Pelvis & $-18.7 \% \pm 5.2 \%(19.4 \%)$ & $-10.8 \% \pm 5.0 \%(11.9 \%)$ \\
& Cerebrum & $-12.5 \% \pm 3.3 \%(13.0 \%)$ & $-9.7 \% \pm 4.1 \%(10.5 \%)$ \\
& Aorta & $-2.8 \% \pm 6.7 \%(7.3 \%)$ & $-3.2 \% \pm 3.9 \%(5.1 \%)$ \\
\hline Lesions (VOI) & Liver & $-5.2 \% \pm 2.8 \%(5.8 \%)$ & $-4.7 \% \pm 2.9 \%(5.5 \%)$ \\
& Soft tissue & $-1.8 \% \pm 4.9 \%(5.3 \%)$ & $-2.5 \% \pm 3.2 \%(4.1 \%)$ \\
\hline Tissue classes & (Near) bone & $-5.2 \% \pm 7.2 \%(8.8 \%)$ & $-4.6 \% \pm 5.2 \%(7.0 \%)$ \\
& (Near) lung & $7.5 \% \pm 14.6 \%(16.4 \%)$ & $3.9 \% \pm 9.2 \%(10.0 \%)$ \\
& Soft & $-1.5 \% \pm 1.8 \%(2.4 \%)$ & $-2.0 \% \pm 1.7 \%(2.6 \%)$ \\
& Fat & $2.4 \% \pm 3.0 \%(3.8 \%)$ & $0.5 \% \pm 1.7 \%(1.7 \%)$ \\
& Bone & $-21.8 \% \pm 2.9 \%(21.9 \%)$ & $-15.3 \% \pm 2.3 \%(15.5 \%)$ \\
& Lung & $-3.4 \% \pm 11.5 \%(11.9 \%)$ & $-2.9 \% \pm 7.1 \%(7.7 \%)$ \\
\hline
\end{tabular}

Data are mean $\pm S D$, followed by RMSE in parentheses. 


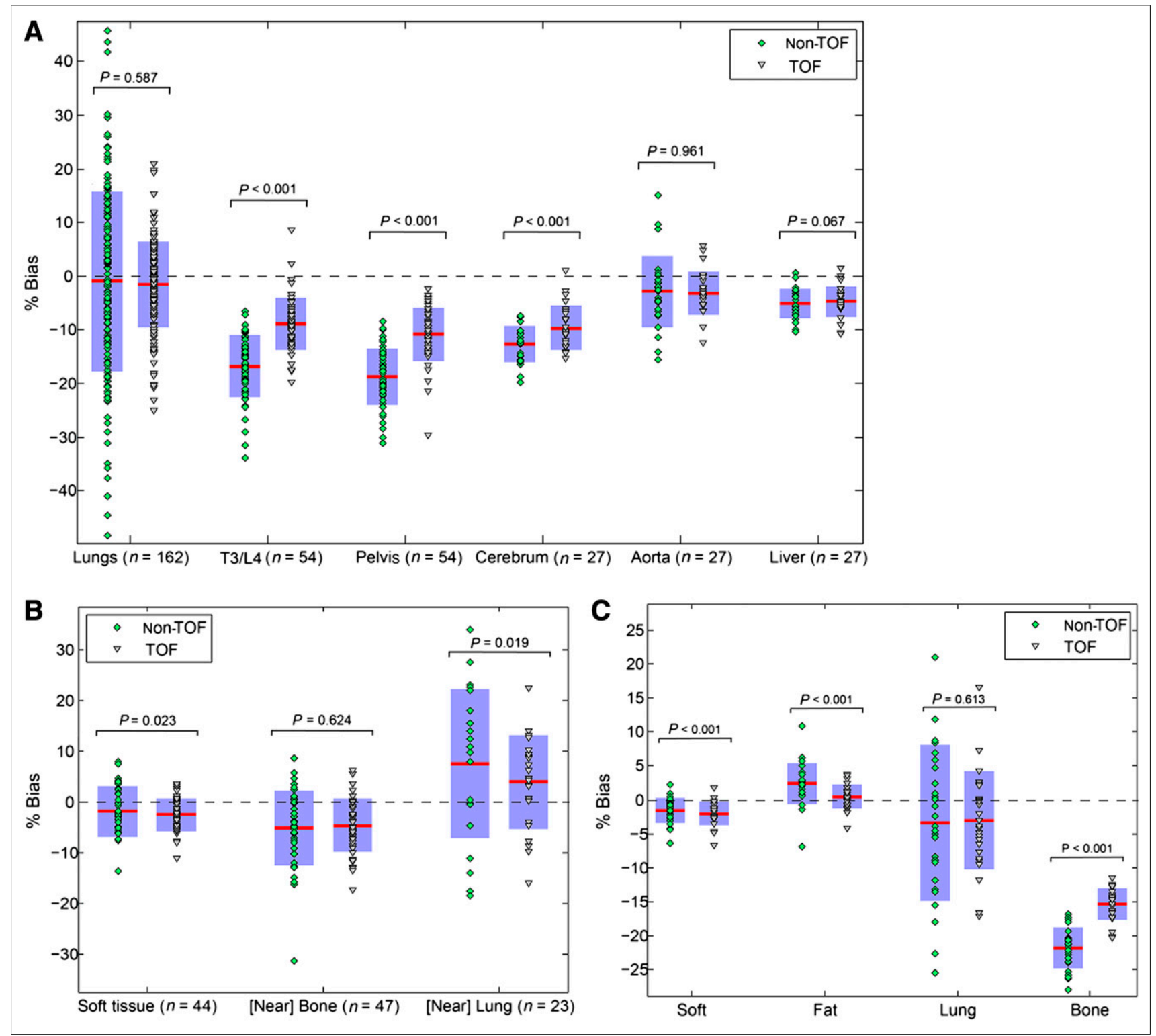

FIGURE 2. Relative errors of SUV mean estimations between MRAC PET and reference CTAC PET images reconstructed with and without TOF in normal tissues (A), lesions (B), and various tissue classes (C). Means and 2 SDs are indicated by horizontal bars and vertical boxes, respectively.

effective TOF resolution of $580 \mathrm{ps}$ and reconstructed using an ordinary Poisson ordered-subset expectation maximization (OSEM) algorithm with point spread function and TOF. The default manufacturerprovided reconstruction parameters for OSEM plus point spread function with and without TOF were 2 iterations and 21 subsets, and 2 iterations and 24 subsets, respectively. The whole-body image matrix size was $200 \times 200$ with $4 \times 4 \times 2 \mathrm{~mm}$ voxels. The CT subsystem is a 128-slice CT scanner (SOMATOM Definition AS+; Siemens Healthcare) with an extended transaxial field of view of $780 \mathrm{~mm}$.

\section{Clinical Studies}

In total, 27 patients ( 13 women, 14 men; average age, $58.8 \pm 18.2$ y) referred for ${ }^{18} \mathrm{~F}-\mathrm{FDG}(n=25)$ and ${ }^{18} \mathrm{~F}$-choline $(n=2)$ PET/CT exanimations were included in this study. The institutional ethics committee approved this retrospective study. The patients had an average body mass index of $25.1 \pm 4.2 \mathrm{~kg} / \mathrm{m}^{2}$. They were injected with a standard dose of ${ }^{18} \mathrm{~F}-\mathrm{FDG}(250.5 \pm 44.4 \mathrm{MBq})$ and ${ }^{18} \mathrm{~F}$-choline $(329.1 \pm 1.2$ $\mathrm{MBq})$. After an uptake time of 60 and $10 \mathrm{~min}$ for ${ }^{18} \mathrm{~F}-\mathrm{FDG}$ and
${ }^{18}$ F-choline administration, whole-body PET/CT FlowMotion scanning (Siemens Healthcare) was performed for 2 scan ranges, from the toes to the mid thigh with a bed speed of $1.1 \mathrm{~mm} / \mathrm{s}$, and from the mid thigh to the vertex with a speed of $0.7 \mathrm{~mm} / \mathrm{s}$. The average acquisition time was $24.9 \pm 5.4 \mathrm{~min}$. A whole-body CT scan protocol was performed for PET attenuation correction using 100-120 kVp, $150 \mathrm{mAs}$, and 5-mm slice thickness.

\section{Simulation Studies}

To explore the limiting impact of TOF on MRAC PET quantification errors, we conducted a series of 3-dimensional analytic simulations with different TOF timing resolutions using an in-house TOF PET simulator, developed for the native geometry of the mCT system using MATLAB (The MathWorks) with single-program multiple-data parallel processing. The software was validated using the experimental National Electrical Manufacturers Association phantom. Effective timing resolutions of 580, 350, and 100 ps were modeled to respectively represent the typical TOF resolution of the first 

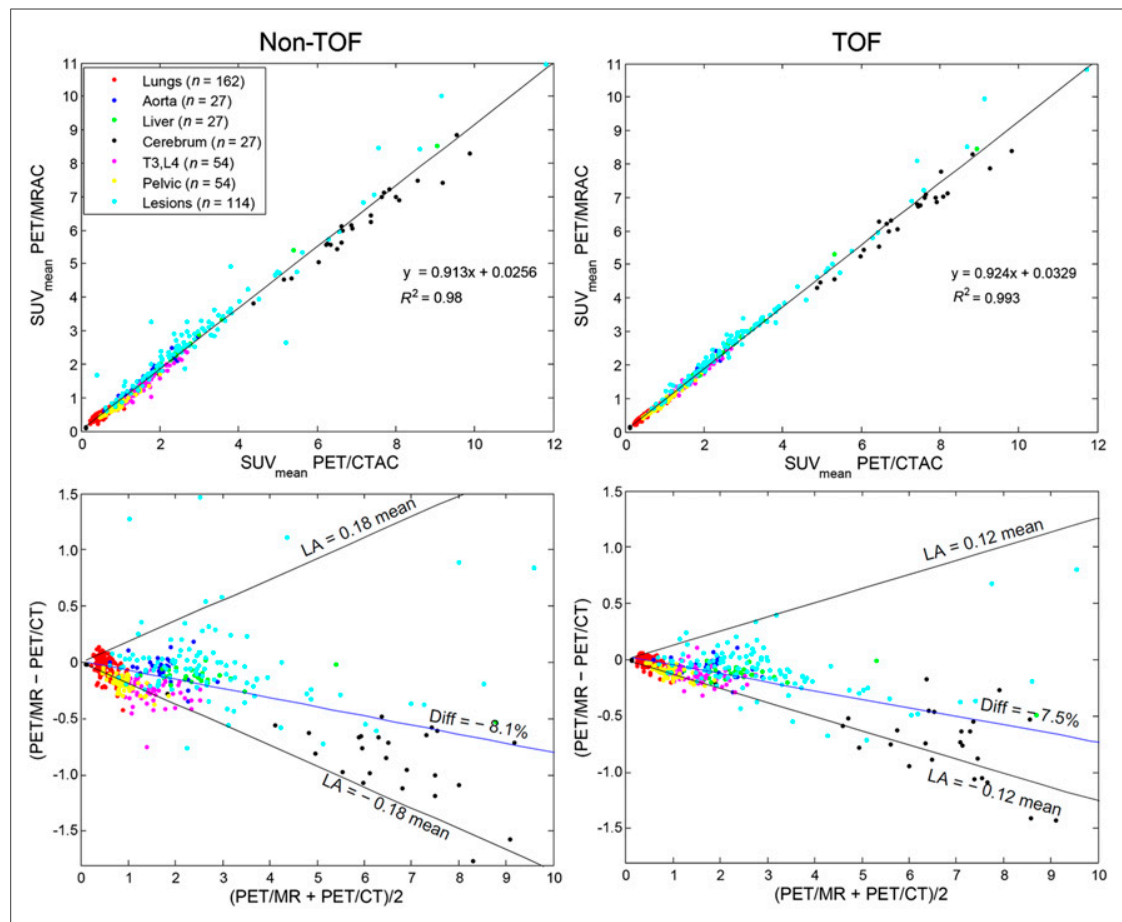

FIGURE 3. (Top) Scatterplots showing results of linear regression analysis between SUV mean in different VOls of MRAC PET and reference CTAC PET with and without TOF. (Bottom) BlandAltman plots of SUV $V_{\text {mean }}$ for MRAC PET and reference CTAC PET with and without TOF. Differences and limits of agreement (LA) are expressed as function of average SUVs of PET/MR and PET/CT.

generation of clinical TOF PET/MR scanners, introduced since 2010 (19); the current generation of silicon photomultiplier-based TOF PET scanners (2); and future-generation PET scanners with the best possible timing resolution currently achievable in the laboratory (18). Realistic whole-body phantoms were derived from clinical ${ }^{18} \mathrm{~F}$-FDG PET/CT studies to simulate the biodistribution of ${ }^{18} \mathrm{~F}$ FDG, the patient-specific CT-based attenuation correction (CTAC) map, and the respiratory-phase mismatch between PET and MRAC images. TOF sinogram data and actual attenuation factors were obtained by forward projection of activity and attenuation maps. Poisson noise realizations were simulated for 80 million counts. The contribution of scattered and random coincidences was ignored. The PET activity maps were reconstructed for 4 overlapping beds with a matrix size of $200 \times 200 \times 109$ per bed, using an ordinary Poisson OSEM algorithm with 3 iterations and 28 subsets for non-TOF, and 3 iterations and 21 subsets for TOF reconstructions.

\section{Attenuation Map Generation}

To derive MRAC maps, CT images of patients were segmented into 4 tissue classes: background air, lung, fat, and nonfat soft tissues. Background air and lungs were segmented using a seeded regiongrowing technique implemented in the itK-SNAP software (20). The fat tissue class was segmented by thresholding CT intensity values between -470 and -53 Hounsfield units (corresponding to attenuation coefficients at $511 \mathrm{keV}$ between 0.05 and $0.095 \mathrm{~cm}^{-1}$ ). The soft-tissue class was then defined as the complement of the segmented classes. In this procedure, bones and air pockets are assigned to soft-tissue class. Mean attenuation coefficients of $0,0.0221,0.0864$, and $0.0975 \mathrm{~cm}^{-1}$ were assigned to background air, lungs, fat, and nonfat soft-tissue classes, respectively. Because of intrinsic differences in image contrast between CT and MR imaging, the resulting 4-class attenuation maps might differ from the scanner's 4-class MRAC maps in tissue content. Supplemental Figure 1 (supplemental materials are available at http://jnm. snmjournals.org) compares both attenuation maps of a patient who underwent a PET/MR imaging scan on the Ingenuity PET/MR scanner (Philips) (19) and a complementary $\mathrm{PET} / \mathrm{CT}$ scan in which excellent coregistration was achieved. As can be seen, both attenuation maps are in good agreement, with sparse differences in fat content.

\section{Data Analysis}

Four PET image reconstructions were performed for each patient: reference CTAC PET and MRAC PET with and without TOF. The relative quantification error (bias) in tracer uptake was calculated on a voxel-by-voxel basis $(i)$ for each patient as follows:

$$
\operatorname{Bias}_{i}^{m}=100 \times \frac{\left(\mathrm{SUV}_{\mathrm{MRAC}}^{m}\right)_{i}-\left(\mathrm{SUV}_{\mathrm{CTAC}}^{\mathrm{m}}\right)_{i}}{\left(\mathrm{SUV}_{\mathrm{CTAC}}^{m}\right)_{i}},
$$

where $m$ is the reconstruction method (nonTOF or TOF). The difference in bias between the non-TOF and TOF methods was then evaluated using volume-of-interest (VOI) and classwise analyses. For each patient, 13 VOIs were defined on normal tissues including the lungs (upper, middle, and lower portions of left and right lungs), liver, aorta, cerebrum, T3, L4, and ilia of pelvis (left and right). Moreover, $80 \%$ isocontour VOIs were defined on the lesion locations in TOF CTAC PET images. Lesions were classified into 3 groups: soft tissue $(n=44)$, bones or near bone $(n=47)$, and lungs or near lung $(n=23)$. For classwise analyses, the original CT images were down-sampled to the resolution of PET images and segmented using region-growing and thresholding techniques into lungs, fat, soft tissue, and bones. The distributions of some VOIs and tissue classes for a representative patient are shown in Supplemental Figure 2. For the defined VOIs, the mean $(\mu)$, SD $(\sigma)$, and root-mean-squared error (RMSE) of bias $\left(\sqrt{\mu^{2}+\sigma^{2}}\right)$ was calculated. The correlation between MRAC PET and CTAC PET with and without TOF was determined on scatterplots using Pearson correlation analysis. The concordance between the SUVs was evaluated using BlandAltman plots. The limits of agreement (LA) were calculated from logarithmically transformed values. The statistical significance of differences in SUV bias was also evaluated using the Wilcoxon signedrank test. The differences were considered statistically significant for $P$ values of less than 0.05 .

\section{RESULTS}

\section{Clinical Studies}

Figure 1 shows the bias maps of PET images reconstructed using non-TOF and TOF MRAC for 2 patients with different body mass indexes. The maps show that the maximum errors occur over the bones, lungs, and air gaps. They are substantially reduced by TOF PET reconstruction. Table 1 summarizes the mean, SD, and RMSE of SUV $_{\text {mean }}$ bias between non-TOF MRAC PET and CTAC PET images, and between TOF MRAC PET and CTAC PET images, in different VOIs located in normal regions and lesions and in defined tissue classes. 


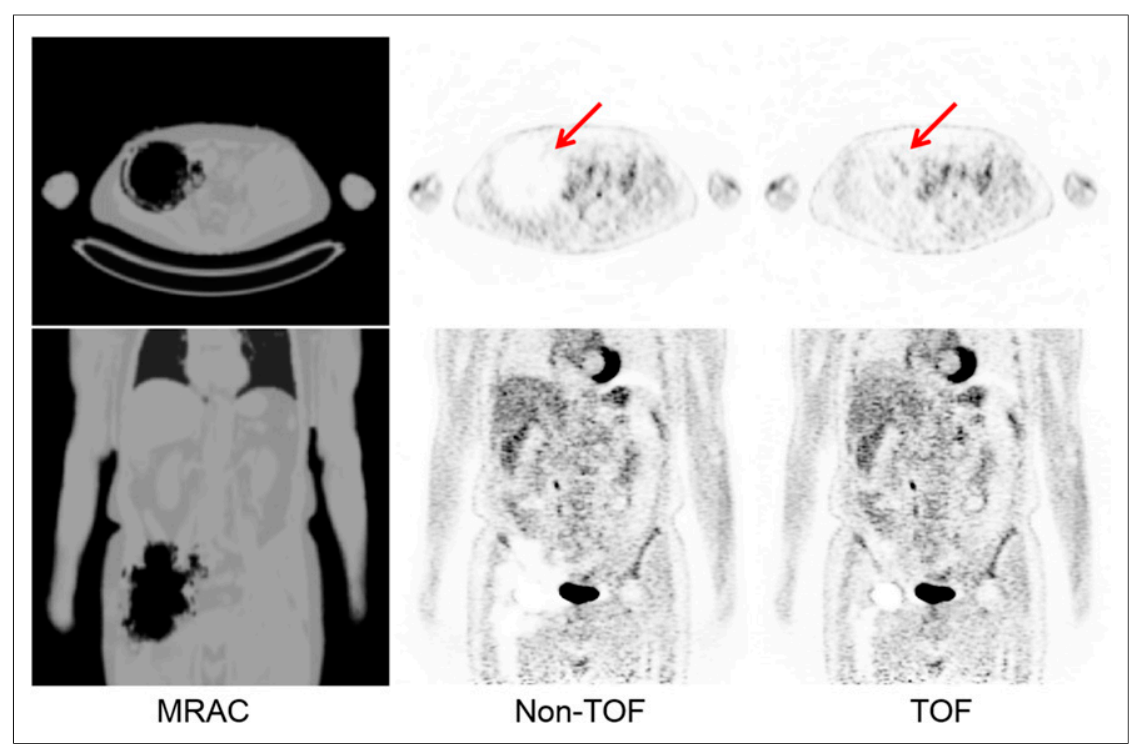

FIGURE 4. Comparison of non-TOF and TOF PET images in presence of metal-induced susceptibility artifacts on MRAC attenuation map.

Figure 2A compares the mean and $\mathrm{SD}$ of $\mathrm{SUV}_{\text {mean }}$ bias between non-TOF and TOF PET images in VOIs defined on different tissues with normal tracer uptake. In this figure, the marker points show the mean of bias in each VOI, whereas the horizontal bars and vertical boxes indicate the mean and 2 SDs of bias between VOIs in each region. For the VOIs defined on the lungs $(n=162)$, the non-TOF MRAC resulted in an underestimation of $-1.0 \% \pm 16.6 \%$ with a RMSE of $16.7 \%$, whereas its TOF counterpart yielded an error of $-1.5 \% \pm 8.0 \%$ with a RMSE of $8.1 \%$ (Table 1 ). For the total VOIs defined on T3, L4, pelvis, and cerebrum $(n=135)$, nonTOF and TOF methods resulted in an average bias error of $-16.7 \% \pm 5.6 \%$ with an RMSE of $17.6 \%$ and $-9.8 \% \pm 4.8 \%$ with an RMSE of $10.9 \%$, respectively. Similarly, for all VOIs defined on soft tissues, aorta, and liver $(n=54)$, non-TOF and TOF methods showed an average bias of $-3.9 \% \pm 5.2 \%$ with $6.5 \%$ RMSE and $-3.4 \% \pm 3.5 \%$ with $5.3 \%$ RMSE, respectively. The statistical analysis of the results revealed that the difference in the bias performance between non-TOF and TOF MRAC methods is significant over the vertebra, pelvis, and cerebrum $(P<0.001)$, whereas there is no proof of statistically significant differences in the lungs $(P=0.587)$, aorta $(P=0.961)$, and liver $(P=0.067)$.

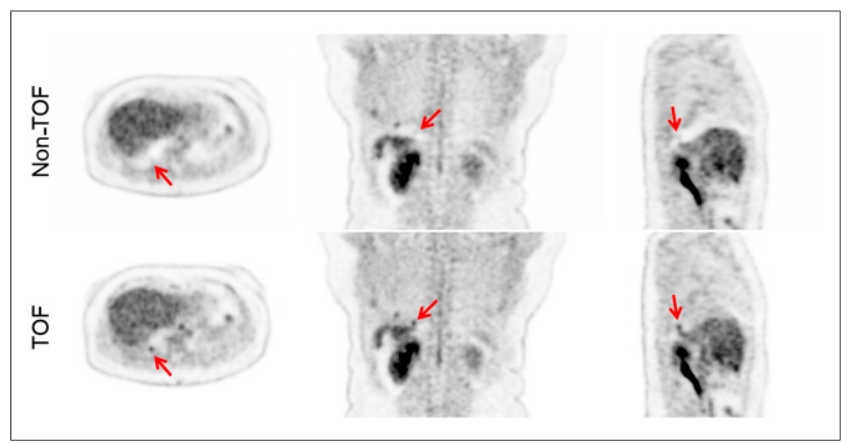

FIGURE 5. Comparison of non-TOF and TOF PET images in presence of respiratory-phase mismatch between MRAC attenuation map and corresponding PET images.
Figure $2 \mathrm{~B}$ shows the mean $\pm \mathrm{SD}$ of bias between non-TOF and TOF PET images in VOIs defined on lesions, grouped into 3 categories depending on their location: soft tissue, for lesions seated in fat and nonfat tissues $(n=44)$; (near) bone, for lesions located on bones or on soft tissues close to bone $(n=47)$; and (near) lungs, for lesions located in the lungs or in the immediate vicinity of the lungs, mainly the mediastinal lymph nodes $(n=23)$. The results presented in Table 1 show that in soft tissue and (near) bone lesions, the non-TOF and TOF MRAC methods result in underestimation of $\mathrm{SUV}_{\text {mean }}$ with comparable RMSE biases of $5.3 \%$ versus $4.1 \%$ and $8.8 \%$ versus $7.0 \%$, respectively. For lesions located in or near the lungs, the methods overestimate $\mathrm{SUV}_{\text {mean }}$. The TOF MRAC method, however, showed an improved performance by achieving a bias of $3.9 \% \pm 9.2 \%$ (10.0\% RMSE) compared with its nonTOF counterpart with a bias of $7.5 \% \pm 14.6 \%$ (16.4\% RMSE). The statistical analysis showed that there is a significant difference in bias performance of the methods in soft tissue $(P=0.023)$ and in or near lung $(P=0.019)$ lesions, whereas there is no proof of statistically significant differences in or near bone lesions $(P=0.624)$.

Figure $2 \mathrm{C}$ compares the mean $\pm \mathrm{SD}$ of bias between non-TOF and TOF methods in nonfat soft-tissue, fat, lung, and bone tissue classes. Each marker point represents the mean of bias in each patient, calculated over all voxels belonging to the tissue classes. According to the results summarized in Table 1, the RMSE bias performance of the 2 methods is less than $5 \%$ in all voxels in softand fat-tissue classes. In the fat-tissue class, both methods overestimate the SUV. However, the TOF MRAC method was found to reduce both the mean and the SD of the bias. Over the bones, an underestimation of $-21.8 \% \pm 2.9 \%(21.9 \%$ RMSE) and $-15.3 \% \pm 2.3 \%$ ( $15.5 \%$ RMSE) was achieved by non-TOF and TOF methods, respectively. Similarly, the methods showed underestimations of $-3.4 \% \pm 11.5 \%(11.9 \%$ RMSE) and $-2.9 \% \pm 7.1 \%$ (7.7\% RMSE) in the lungs, respectively. TOF PET reconstruction resulted in a significant difference in all tissue classes $(P<0.001)$ except the lungs $(P=0.613)$.

The MRAC PET images were further analyzed for SUV correlation and concordance with respect to CTAC PET images. Figure 3 (top) shows the scatterplots of $\mathrm{SUV}_{\text {mean }}$ in all normal tissues and lesions in PET images reconstructed using MRAC and CTAC with and without TOF, with correlation coefficients and corresponding regression equations. Overall, there is a good correlation between non-TOF MRAC and non-TOF CTAC SUVs, with $R^{2}=0.980$, which is improved by TOF reconstruction $\left(R^{2}=0.993\right)$. Figure 3 (bottom) shows the Bland-Altman concordance analysis of the MRAC methods. Differences and LA are expressed as a function of average SUVs of PET/MR and PET/CT. The regression lines of the difference (indicated with percentage slope) shows a systematic underestimation of SUV for both nonTOF and TOF MRAC methods ( $-8.1 \%$ and $-7.5 \%$, respectively). The results show that the TOF reconstruction can reduce the bias and the SD of bias. 


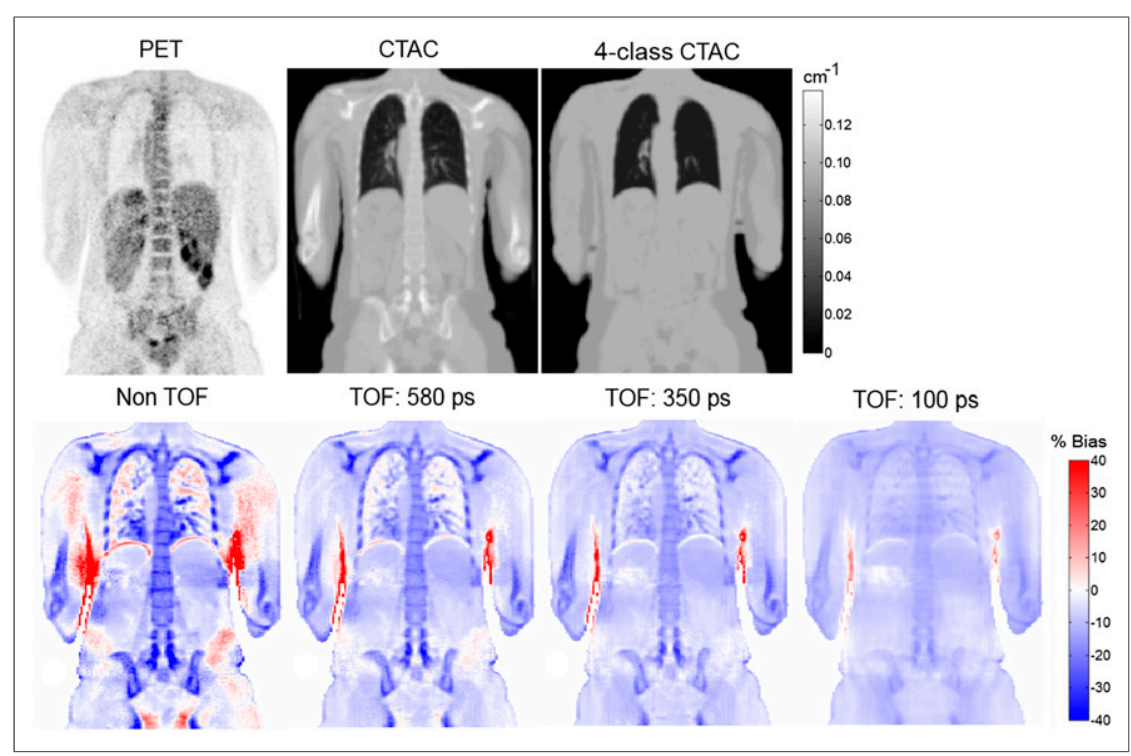

FIGURE 6. Simulation of impact of TOF reconstruction on PET quantification errors of MRAC method. (Top) PET activity, CTAC, and 4-class MRAC maps derived from whole-body ${ }^{18}$ F-FDG PET scan. (Bottom) Bias maps between MRAC PET and CTAC PET images for different timing resolutions.

\section{Simulation Studies}

Figure 6 shows the PET, actual CTAC, and MRAC images used in our non-TOF and TOF simulations, together with the bias maps corresponding to non-TOF and TOF images with timing resolutions of 580, 350, and 100 ps. For each simulated TOF resolution, 2 PET images were reconstructed (using reference CTAC and 4-class MRAC maps). The results clearly show that PET quantification errors induced by the 4-class maps are reduced as the timing resolution is improved, especially over the bones. Classwise analyses showed that over bones, the non-TOF reconstruction results in an underestimation of $-25.15 \% \pm 9.8 \% \quad(27.0 \%$ RMSE), which is reduced to $-19.3 \% \pm$ $6.66 \%$ (20.42\% RMSE), $-16.63 \% \pm 5.4 \%$ $(17.51 \%$ RMSE), and $-12.47 \% \pm 3.23 \%$ (12.88\% RMSE) using TOF reconstructions with timing resolutions of 580, 350, and $100 \mathrm{ps}$, respectively. For the non-TOF and the TOF with 580-, 350-, and 100-ps resolutions, RMSEs of bias averaged over all tissue

Figure 4 compares the non-TOF and TOF PET images of a patient presenting with metal-induced susceptibility artifacts in MRAC maps. The patient had a PET/MR imaging and complementary PET/CT scan. The MR and CT images were nonrigidly registered. The void regions induced by the metal implant were then transferred to the derived 4-class CTAC map. As can be seen, the TOF reconstruction can substantially reduce the artifacts close to the hip implant. Figure 5 compares PET images of a patient with respiratory-phase mismatch between PET and MRAC maps. As pointed out by the arrows, one of the lesions is indiscernible in non-TOF PET image because of the undercorrection caused by respiratory-phase mismatch artifacts. However, the TOF reconstruction has effectively suppressed the artifacts, thus improving lesion detectability. classes except bones were $13.11 \%, 9.15 \%, 8.61 \%$, and $8.28 \%$, respectively. The impact of TOF reconstruction on respiratory-phase mismatch artifacts was further evaluated in a simulated clinical ${ }^{18}$ F-FDG scan (Fig. 7). A respiratory-phase mismatch was simulated between the actual CTAC and MRAC maps with 12-mm displacement of the diaphragm as if the MR images were acquired at the end of inspiration. A 4-mm spheric tumor was also inserted in the PET image to simulate a case in which respiratory-phase mismatch impaired the detectability of a liver lesion. As shown in the figure, the activity of the tumor and superior border of liver was suppressed by white-band banana artifacts. As the timing resolution improves, the tumor and liver lobe become more discernible and lean toward their actual uptake. The bias maps also demonstrate that improved TOF resolution reduces quantification errors.

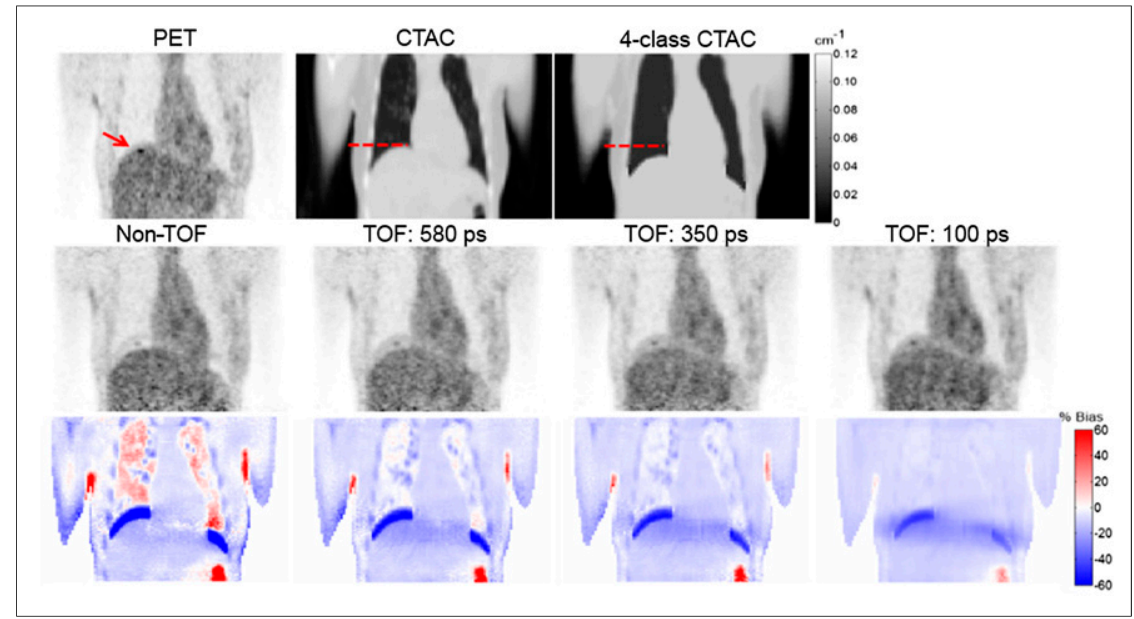

FIGURE 7. Simulation of impact of TOF reconstruction on reduction of artifacts induced by respiratory-phase mismatch between PET and MRAC images. (Top) PET activity, CTAC, and 4-class MRAC maps derived from whole-body ${ }^{18}$ F-FDG PET scan. (Middle) PET image reconstructed using different TOF timing resolutions. (Bottom) Corresponding bias maps between MRAC PET and CTAC PET images.

\section{DISCUSSION}

To the best of our knowledge, this was the first clinical study highlighting the impact and importance of TOF in MRAC PET image reconstruction. Using VOIbased and classwise analyses, we demonstrated that TOF can reliably compensate for erroneous MRAC maps, especially over the bone and lungs, where the maximum errors occur. The results of 135 VOIs defined in or near bone regions with normal tracer uptake showed an average underestimation of $16.7 \%$ and $9.8 \%$ for non-TOF and TOF MRAC reconstructions, respectively. Classwise evaluations showed that these methods result in maximum underestimations of $21.8 \%$ and $15.3 \%$, respectively, over the bones. Our non-TOF results are consistent with the more than $15 \%$ errors previously reported (9) using 
PET/MR datasets. The VOI-based evaluation of 47 lesions in or near bone showed an average $5 \%$ error for both methods, which is in agreement with the $8 \%$ and $6 \%$ errors reported previously $(5,14)$. Consistent with previous observations $(5,11)$, the results of 164 VOIs in normal tissues of the lungs showed an average SUV underestimation of less than $2 \%$ for non-TOF and TOF methods. However, non-TOF MRAC showed a large SD (16.6\%), which was substantially reduced by TOF $(8.1 \%)$. The reason for this large SD is that the standard MRAC methods do not take the intra- or interpatient variability of lung attenuation coefficients into account and assign a constant value for the lungs in all patients. Our results showed that non-TOF MRAC results in $7.5 \%$ overestimation of lesions in or near the lungs and that this overestimation is reduced to $3.9 \%$ by TOF image reconstruction. For soft-tissue lesions, the results showed that both methods give rise to errors of less than $5 \%$, which is in agreement with previous studies $(5,14)$.

Our study also demonstrated a strong correlation between PET/ MR and PET/CT SUVs in normal tissues and lesions. It was found that using TOF PET image reconstruction, the dispersion of data points around the regression line is reduced, especially for lesions, resulting in a higher coefficient of determination. The Bland-Altman plots revealed a limited concordance between PET/MR and PET/CT measurements, in particular for lesions with high SUVs. As a result, both non-TOF and TOF methods showed a systematic bias, proportional to average SUVs. Nonetheless, the limit-of-agreement results showed that TOF can reduce the SD of errors.

In this study, we used the default vendor-provided reconstruction parameters of $2 / 24$ and 2/21 iterations/subsets for the non-TOF and TOF reconstructions, respectively. Indeed, the non-TOF reconstruction did not result in the same convergence rate achieved by TOF reconstruction. Achieving the same convergence rate for lesions using both reconstruction methods is barely accomplished in the clinical setting, since it depends on the size and location of the lesions and lesion-to-background ratio, which varies for different lesions and patients. However, we evaluated SUV quantification errors of nonTOF and TOF MRAC methods using different iterations for one clinical study. As shown in Supplemental Figure 3, the bias will change with iteration number, especially in bones with non-TOF reconstruction. The results show that the bias introduced by TOF MRAC is almost steady for all tissue classes except bones. Consequently, the TOF MRAC method resulted in significantly less bias than its non-TOF counterpart for each iteration. Our simulation results demonstrated that as TOF timing resolution improves toward $100 \mathrm{ps}$, targeted for future TOF PET/MR scanners, SUV quantification errors induced by inaccurate MRAC maps are substantially reduced, thereby improving the level of accuracy of PET quantification.

\section{CONCLUSION}

In this work, we evaluated the impact of TOF PET image reconstruction on SUV quantification errors caused by standard MR segmentation-based attenuation correction of PET data using simulation and clinical studies. Our results demonstrated that TOF capability can substantially reduce the mean and SD of bias over the lungs and bones. Non-TOF MRAC resulted in $11.9 \%$ and $21.9 \%$ root-mean-squared errors in the lungs and bones, respectively. These errors were reduced to $7.7 \%$ and $15.5 \%$, respectively, when TOF was used. The results showed that the root-mean-square errors of non-TOF and TOF methods in soft-tissue and fat regions are less than $5 \%$. From a clinical perspective, our study suggests that as the timing resolution of TOF PET/MR scanners improves, PET quantitative accuracy for bone and lung lesions increases.

\section{DISCLOSURE}

The costs of publication of this article were defrayed in part by the payment of page charges. Therefore, and solely to indicate this fact, this article is hereby marked "advertisement" in accordance with 18 USC section 1734. This work was supported in part by the Swiss National Science Foundation under grant SNSF 31003A149957 and the Indo-Swiss Joint Research Programme ISJRP138866. No other potential conflict of interest relevant to this article was reported.

\section{REFERENCES}

1. Torigian DA, Zaidi H, Kwee TC, et al. PET/MR imaging: technical aspects and potential clinical applications. Radiology. 2013;267:26-44.

2. Levin C, Glover G, Deller T, McDaniel D, Peterson W, Maramraju SH. Prototype time-of-flight PET ring integrated with a 3 T MRI system for simultaneous whole-body PET/MR imaging [abstract]. J Nucl Med. 2013;54(suppl 2):148.

3. Zaidi H. Is MRI-guided attenuation correction a viable option for dual-modality PET/MR imaging? Radiology. 2007;244:639-642.

4. Keller SH, Holm S, Hansen A, et al. Image artifacts from MR-based attenuation correction in clinical, whole-body PET/MRI. MAGMA. 2013;26:173-181.

5. Martinez-Möller A, Souvatzoglou M, Delso G, et al. Tissue classification as a potential approach for attenuation correction in whole-body PET/MRI: evaluation with PET/CT data. J Nucl Med. 2009;50:520-526.

6. Hofmann M, Bezrukov I, Mantlik F, et al. MRI-based attenuation correction for whole-body PET/MRI: quantitative evaluation of segmentation- and atlas-based methods. J Nucl Med. 2011;52:1392-1399.

7. Salomon A, Goedicke A, Schweizer B, Aach T, Schulz V. Simultaneous reconstruction of activity and attenuation for PET/MR. IEEE Trans Med Imaging. 2011;30:804-813.

8. Mehranian A, Zaidi H. Joint estimation of activity and attenuation in TOF-PET/ MR using constrained Gaussian mixture models [abstract]. J Nucl Med. 2014;55 (suppl 1):645.

9. Bezrukov I, Schmidt H, Mantlik F, et al. MR-based attenuation correction methods for improved PET quantification in lesions within bone and susceptibility artifact regions. J Nucl Med. 2013;54:1768-1774.

10. Ouyang J, Se Young C, Petibon Y, Bonab AA, Alpert N, El Fakhri G. Bias atlases for segmentation-based PET attenuation correction using PET-CT and MR. IEEE Trans Nucl Sci. 2013;60:3373-3382.

11. Arabi H, Rager O, Alem A, Varoquaux A, Becker M, Zaidi H. Clinical assessment of MR-guided 3-class and 4-class attenuation correction in PET/MR. Mol Imaging Biol. August 16, 2014 [Epub ahead of print].

12. Keereman V, Holen RV, Mollet P, Vandenberghe S. The effect of errors in segmented attenuation maps on PET quantification. Med Phys. 2011;38:6010-6019.

13. Samarin A, Burger C, Wollenweber S, et al. PET/MR imaging of bone lesions: implications for PET quantification from imperfect attenuation correction. Eur J Nucl Med Mol Imaging. 2012;39:1154-1160.

14. Schulz V, Torres-Espallardo I, Renisch S, et al. Automatic, three-segment, MRbased attenuation correction for whole-body PET/MR data. Eur J Nucl Med Mol Imaging. 2011;38:138-152.

15. Catana C, van der Kouwe A, Benner T, et al. Toward implementing an MRIbased PET attenuation-correction method for neurologic studies on the MR-PET brain prototype. J Nucl Med. 2010;51:1431-1438.

16. Conti $\mathrm{M}$. Why is TOF PET reconstruction a more robust method in the presence of inconsistent data? Phys Med Biol. 2011;56:155-168.

17. Boellaard R, Hofman MB, Hoekstra OS, Lammertsma AA. Accurate PET/MR quantification using time of flight MLAA image reconstruction. Mol Imaging Biol. 2014;16:469-477.

18. van Dam HT, Borghi G, Seifert S, Schaart DR. Sub-200 ps CRT in monolithic scintillator PET detectors using digital SiPM arrays and maximum likelihood interaction time estimation. Phys Med Biol. 2013;58:3243-3257.

19. Zaidi H, Ojha N, Morich M, et al. Design and performance evaluation of a whole-body Ingenuity TF PET-MRI system. Phys Med Biol. 2011;56:30913106.

20. Yushkevich PA, Piven J, Hazlett HC, et al. User-guided 3D active contour segmentation of anatomical structures: significantly improved efficiency and reliability. Neuroimage. 2006;31:1116-1128. 